Research Article

\title{
Genetic Analysis of 25 Patients with $5 \alpha$-Reductase Deficiency in Chinese Population
}

\author{
Bing Han, ${ }^{1}$ Tong Cheng, ${ }^{1}$ Hui Zhu, ${ }^{1}$ Jie Yu, ${ }^{1}$ Wen-jiao Zhu, ${ }^{1}$ Huai-dong Song, ${ }^{2}$ \\ Haijun Yao $\mathbb{D}^{3},{ }^{3}$ and Jie Qiao $\mathbb{D}^{1}$ \\ ${ }^{1}$ Department of Endocrinology, Shanghai Ninth People's Hospital, Shanghai Jiao Tong University School of Medicine, \\ Shanghai, China \\ ${ }^{2}$ Central Laboratory, Clinical Research Center, Shanghai Ninth People's Hospital, Shanghai Jiao Tong University School of Medicine, \\ Shanghai, China \\ ${ }^{3}$ Department of Urology, Shanghai Ninth People's Hospital, Shanghai Jiao Tong University School of Medicine, Shanghai, China
}

Correspondence should be addressed to Haijun Yao; dryaohj@163.com and Jie Qiao; qiaoj2001@126.com

Received 4 November 2019; Revised 6 February 2020; Accepted 2 March 2020; Published 9 June 2020

Academic Editor: Sarah H. Elsea

Copyright $(2020$ Bing Han et al. This is an open access article distributed under the Creative Commons Attribution License, which permits unrestricted use, distribution, and reproduction in any medium, provided the original work is properly cited.

Background. A deficiency in steroid $5 \alpha$-reductase type 2 is an autosomal recessive disorder. Affected individuals manifested ambiguous genitalia, which is caused by decreased dihydrotestosterone (DHT) synthesis in the fetus. Methods. We analyzed 25 patients with $5 \alpha$-reductase deficiency in China. Seventeen of the 25 patients (68\%) were initially raised as females. Sixteen patients changed their social gender from female to male after puberty. Results. Eighteen mutations were identified in these patients. p.Gly203Ser and p.Gln6* were found to be the most prevalent mutations. On the basis of the genotype of these patients, we divided them into different groups. There was no significant difference in hormone levels and external masculinization score (EMS) in patients with or without these prevalent mutations. Twelve common single-nucleotide polymorphisms (SNPs) near the p.Gln6* mutation were chosen for haplotype analysis. Three haplotypes were observed in 6 patients who had the p.Gln6* mutation (12 alleles). Conclusion. We analyzed mutations of the SRD5A2 gene in Chinese patients with $5 \alpha$-reductase deficiency. Although hotspot mutations exist, no founder effect of prevalent mutations in the SRD5A2 gene was detected in the Chinese population.

\section{Introduction}

$S R D 5 A 2$, the gene encoding steroid $5 \alpha$-reductase type 2 $(5 \alpha-\mathrm{RD} 2)$, is located on chromosome $2 \mathrm{p} 23$ and contains 5 exons [1]. $5 \alpha$-RD2 contains 254 amino acids and is predominantly expressed in fetal genital skin, male productive tissues, and the prostate [2]. It converts testosterone (T) to dihydrotestosterone (DHT), which plays a vital role in the development of male external genitalia [3].

$5 \alpha$-RD2 deficiency is an autosomal recessive disorder caused by mutations in the SRD5A2 gene that impair the conversion of T to DHT. Since first described in 1974 $[4,5]$, more than 100 mutations of SRD5A2 have been identified worldwide [6-8]. Phenotypes of $5 \alpha$-RD2 deficiency range from female external genitalia to hypospadias with microphallus to apparently normal male external genitalia. After puberty, these patients showed different degrees of virilization and often change their social gender from female to male [9].

In some cases, $5 \alpha$-RD2 deficiency shared the same clinical phenotype with androgen insensitivity syndrome, which may have caused misdiagnosis of this disease. Elevated baseline and hCG-stimulated T/DHT ratio is useful to diagnose this disease [10-12]. However, more accurate diagnoses rely on genetic testing, especially in prepubertal patients [13].

In this study, we analyzed 25 Chinese patients with $5 \alpha$-RD2 deficiency. Eighteen mutations in the SRD5A2 gene were identified from these patients. In these mutations, 
p.Gln6* was widely reported. Therefore, we chose 12 SNPs near p.Gln $6 *$ and performed haplotype analysis to determine whether this mutation has a founder effect in the Chinese population.

\section{Patients and Methods}

2.1. Patients. Twenty-five patients, aged from 4 days to 34 years (diagnosis age), with 46,XY DSD were included in this study. Patients 1-14 were reported by Cheng et al. [14]. Patients 15-25 were reported by Zhu et al. [9]. Patients 4 and 17 came from consanguineous families. Patients 8 and 9 were twin brothers with similar phenotypes, whereas patients 21 and 22 were siblings with different phenotypes. The clinical and definitive diagnosis of steroid $5 \alpha$-reductase- 2 deficiency was described previously [9]. External masculinization score (EMS) was also accessed as previously described [15]. This study was approved by the Ethics Committee of our institute. Written informed consent was obtained from all the adult patients themselves or from the parents of patients who were children.

2.2. Mutation Analysis. Genomic DNA of the patients was extracted from peripheral blood leukocytes using a kit (TIANGEN Biotech, Beijing, China). All five exons of the SRD5A2 gene were amplified as previously described [9]. Then, the PCR products were purified by shrimp alkaline phosphatase (SAP, USB, Cleveland, USA) and directly sequenced in both directions. When a novel mutation was found, PCR fragments amplified from the genomic DNA of 100 healthy subjects were also analyzed to exclude polymorphism.

2.3. Haplotype Analysis. For haplotyping analysis, 12 common single-nucleotide polymorphisms (SNPs) near the location of p.Gln6* were selected from the SRD5A2 gene based on the genotyping data from 1468 Chinese Han individuals in a genome-wide association study (GWAS) [16] (rs12470143: SRD5A2 RefSeq Gene NG_ 008365.1:g.47484G>A; rs12470196: SRD5A2 RefSeq Gene NG_008365.1:g.47290G>A; rs57971483: SRD5A2 RefSeq Gene NG_008365.1:g.45532A>G; rs4952220: SRD5A2 RefSeq Gene NG_008365.1:g.45486G>A; rs2300697: SRD5A2 RefSeq Gene NG_008365.1:g.24405G>A; rs2300698: SRD5A2 RefSeq Gene NG_008365.1:g.24249T>C; rs2300699: SRD5A2 RefSeq Gene NG_008365.1:g.24075C>A; rs2300700: SRD5A2 RefSeq Gene NG_008365.1:g.24050C>T; rs2300701: SRD5A2 RefSeq Gene NG_008365.1:g.24034T>C; rs522638: SRD5A2 RefSeq Gene NG_008365.1:g.5367T>C; rs523349: SRD5A2 RefSeq Gene NG_008365.1:g.5336C>G; rs632148: SRD5A2 RefSeq Gene NG_008365.1:g.5010G>C) (http://www.ncbi.nlm.nih .gov/SNP/). Two twin brothers (patients 8 and 9) had the same genotype. Therefore, six patients carrying the p.Gln6* heterozygous mutation were genotyped for these 12 SNPs by PCR and Sanger sequencing. PHASE 2.1 software was used to perform the haplotyping analysis. We also compared the difference in haplotypes between healthy controls and our patients.

2.4. Statistical Analysis. Data were expressed as the means \pm SD. Statistical analysis was determined using the unpaired
Student's $t$-test. The analyses were performed using SPSS version 11.0. $P<0.05$ was considered significant.

\section{Results}

3.1. Clinical Characteristics. The molecular analysis results of 25 patients with $5 \alpha$-RD2 deficiency are summarized in Table 1 . The detailed clinical characteristics were described previously $[9,14]$. Seventeen of the 25 patients $(68 \%)$ were initially raised as females. Among them, 16 patients (94.1\%) had changed their social gender from female to male. Six patients were prepubertal (patients 10,11,15,16,17, and 18 ), and only one (patient 15) was raised as a male before the first examination. Nineteen patients were diagnosed at the age of more than 16 years old. Patients 21 and 22 were siblings but presented with different phenotypes. Isolated micropenis or normal female genitalia were found in patients 16 and 22. The topical application of DHT gel was used by patients $3,4,5,10,16$, and 20 with different diagnostic ages. Six patients were from Guizhou province and four patients were from Jiangxi province. Of the seven patients with p.Gln $6 *$ mutation, three were from Guizhou province and the other four patients were from Sichuan, Anhui, Hubei, and Shandong provinces.

3.2. DNA Sequencing. Eighteen different mutations were identified in 25 patients from 23 unrelated families (Table 1). Compound heterozygous mutations were found in 15 patients, and homozygous mutations were found in 7 patients. The most frequent mutations in our study was p.Gly203Ser and p.Gln $6 *$, found on one allele of 8 (patients $2,4,10,16$, 20, 21, 22, and 25) and 7 patients (patients 1, 6, 8, 9, 11, 12, and 25). The reported polymorphism, p.Val89Leu, was identified in 14 patients.

3.3. Comparison of Hormone Levels and EMS between Different Mutation Types. To investigate the influence of "hotspot" mutations p.Gly203Ser and p.Gln6* on hormone levels, we compared patients with these two mutations and those without the two mutations. In a comparison of 8 patients carrying the p.Gly203Ser mutation and 17 patients carrying other mutations, LH, FSH, T, DHT, T/DHT, and EMS showed no significant difference $(P \geq 0.05)$ in Table 2 . We also compared 7 patients carrying the p.Gln $6 *$ mutation and 18 patients without the p.Gln6* mutation. The results indicated that LH, FSH, T, DHT, and T/DHT were not significantly different $(P \geq 0.05)$. However, EMS showed marginal difference between two groups $(P=0.05)$ (Table 3$)$.

3.4. Haplotype Analysis. The heterozygous nonsense mutation p.Gln $6 *$, which was the most frequent mutation in this study, was identified in 7 cases from 6 pedigrees $(7 / 25$, $28 \%$ ). To identify potential common ancestors for those subjects who carried this mutation, twelve SNPs in and around exon 1 of SRD5A2 spanning $26 \mathrm{~kb}$ were chosen for analysis. Three haplotypes were observed in 6 patients (12 alleles), with CCTCCAGGAAGC and TTCATGTAGGCG in 5 alleles, respectively, whereas CCTCTGTGGGCG were found in 2 alleles. It cannot be concluded that the p.Gln $6 *$ mutation has a founder effect in the Chinese population since in 6 
TABLE 1: Clinical and genetic characteristics of the patients.

\begin{tabular}{|c|c|c|c|c|c|}
\hline Patient no. & Location & Age of diagnose & Sex of rearing at birth & EMS & SRD5A2 mutation \\
\hline 1 & Sichuan & $22 \mathrm{yr}$ & $\mathrm{F}$ to $\mathrm{M}$ & 3 & p.Gln6*/p.Lys35Asn/p.Phe234Leu \\
\hline 2 & Guizhou & $23 \mathrm{yr}$ & M & 6 & p.Gly203Ser/p.Arg227Gln/p.Gly34Arg \\
\hline 3 & Sichuan & $20 \mathrm{yr}$ & M & 5 & p.Leu20Pro/p.Arg246Gln \\
\hline 4 & Jiangxi & $19 \mathrm{yr}$ & M & 6 & p.Gly203Ser/p.Gly203Ser \\
\hline 5 & Liaoning & $23 \mathrm{yr}$ & $\mathrm{F}$ to $\mathrm{M}$ & 2 & p.Ala228Val/- \\
\hline 6 & Guizhou & $24 \mathrm{yr}$ & $\mathrm{F}$ & 2 & p.Gln $6 * /-$ \\
\hline 7 & - & $30 \mathrm{yr}$ & M & 2 & p.Tyr136*/p.Tyr136* \\
\hline 8 & Guizhou & $18 \mathrm{yr}$ & F to $\mathrm{M}$ & 2 & p.Gln6*/p.His162Pro \\
\hline 9 & Guizhou & $18 \mathrm{yr}$ & F to $\mathrm{M}$ & 2 & p.Gln6*/p.His162Pro \\
\hline 10 & Guizhou & $5 \mathrm{yr}$ & F to $\mathrm{M}$ & 2 & p.Gly203Ser/p.Gly203Ser \\
\hline 11 & Anhui & $11 \mathrm{yr}$ & $\mathrm{F}$ to $\mathrm{M}$ & 2 & p.Gln6*/p.Arg227Gln \\
\hline 12 & Hubei & $23 \mathrm{yr}$ & $\mathrm{F}$ to $\mathrm{M}$ & 2 & p.Gln6*/p.Asn193Ser \\
\hline 13 & Yunnan & $18 \mathrm{yr}$ & M & 5 & p.Arg171Ser/p.Gly196Val \\
\hline 14 & Guizhou & $34 \mathrm{yr}$ & $\mathrm{F}$ to $\mathrm{M}$ & 3 & p.Leu20Pro/p.Arg227* \\
\hline 15 & Jiangxi & $4 \mathrm{yr}$ & M & 9 & p.Val89Leu/c.655del \\
\hline 16 & Jiangsu & $4 \mathrm{~d}$ & $\mathrm{~F}$ to $\mathrm{M}$ & 3 & p.Gly203Ser/p.Arg227Gln \\
\hline 17 & Henna & $14 \mathrm{yr}$ & $\mathrm{F}$ to $\mathrm{M}$ & 2.5 & c. $698+2 \mathrm{~T}>\mathrm{C} / \mathrm{c} .698+2 \mathrm{~T}>\mathrm{C}$ \\
\hline 18 & Zhejiang & $12 \mathrm{yr}$ & $\mathrm{F}$ to $\mathrm{M}$ & 2.5 & p.Arg246Gln/p.Arg246Gln \\
\hline 19 & Anhui & $18 \mathrm{yr}$ & $\mathrm{F}$ to $\mathrm{M}$ & 2.5 & p.Arg246Gln/p.Asn193Ser \\
\hline 20 & Jiangxi & $17 \mathrm{yr}$ & $\mathrm{F}$ to $\mathrm{M}$ & 3 & p.Gly203Ser/p.Arg246Gln \\
\hline 21 & - & $18 \mathrm{yr}$ & M & 9 & p.Gly203Ser/c.182-2A>G \\
\hline 22 & - & $23 \mathrm{yr}$ & $\mathrm{F}$ to $\mathrm{M}$ & 3 & p.Gly203Ser/c.182-2A>G \\
\hline 23 & Jiangxi & $16 \mathrm{yr}$ & $\mathrm{F}$ to $\mathrm{M}$ & 3 & p.Ala228Val/p.Ala228Val \\
\hline 24 & Henan & $23 \mathrm{yr}$ & $\mathrm{F}$ to $\mathrm{M}$ & 2.5 & p.Ala228Val/p.Ala228Val \\
\hline 25 & Shandong & $16 \mathrm{yr}$ & $\mathrm{F}$ to $\mathrm{M}$ & 2 & p.Gln6*/p.Gly203Ser \\
\hline
\end{tabular}

F: female; M: male; EMS: external masculinization score. — indicates no mutation in another chromosome.

TABLE 2: Comparison of hormone levels between patients with and without p.GLY203SER mutation.

\begin{tabular}{lccc}
\hline & GLY203SER $(n=8)$ & Other variants $(n=17)$ & $P$ value \\
\hline LH & $6.58 \pm 4.24$ & $8.82 \pm 6.00$ & 0.36 \\
FSH & $5.94 \pm 3.03$ & $14.36 \pm 11.09$ & 0.05 \\
T & $19.27 \pm 12.97$ & $20.74 \pm 11.25$ & 0.77 \\
DHT & $0.56 \pm 0.37$ & $0.49 \pm 0.28$ & 0.61 \\
T/DHT & $40.28 \pm 26.10$ & $52.05 \pm 34.17$ & 0.41 \\
EMS & $4.25 \pm 2.49$ & $3.06 \pm 1.80$ & 0.19 \\
\hline
\end{tabular}

EMS: external masculinization score.

alleles carrying the p.Gln6* mutation, two different haplotypes were found (haplotype 1 and haplotype 3 ), and haplotype 1 was also found to be common in 1494 normal alleles (Table 4).

\section{Discussion}

DHT, converted from testosterone in the skin of the fetal labioscrotal folds as a paracrine factor, induces labioscrotal fusion, the development of the phallic urethra, and phallic
TABLE 3: Comparison of hormone levels between patients with and without p.GLN6* mutation.

\begin{tabular}{lllc}
\hline & GLN6* $(n=7)$ & Other variants $(n=18)$ & $P$ value \\
\hline LH & $8.47 \pm 6.53$ & $7.91 \pm 5.21$ & 0.83 \\
FSH & $16.35 \pm 12.47$ & $9.85 \pm 8.65$ & 0.15 \\
T & $20.25 \pm 12.29$ & $20.28 \pm 11.65$ & 1.00 \\
DHT & $0.35 \pm 0.19$ & $0.57 \pm 0.33$ & 0.15 \\
T/DHT & $64.03 \pm 41.28$ & $42.28 \pm 26.49$ & 0.15 \\
EMS & $2.14 \pm 0.38$ & $3.94 \pm 2.25$ & 0.05 \\
\hline
\end{tabular}

EMS: external masculinization score.

enlargement [17]. Two $5 \alpha$-reductases in humans, encoded by different genes on chromosome 5 and chromosome 2 (SRD5A1 and SRD5A2), were identified to catalyze the conversion of testosterone to DHT. Both enzymes are hydrophobic and membrane bound [1] and have proven difficult to solubilize in active form. With a similar gene structure of 5 exons separated by 4 introns, SRD5A2 was found to be predominantly expressed in the male urogenital tract and prostate and in female genital skin, whereas SRD5A1 is predominantly expressed in the ovary, testis, liver, and 
TABLE 4: Frequencies of the haplotypes with p.Gln6* mutation in 12 cases and 1468 controls.

\begin{tabular}{|c|c|c|c|c|c|c|c|}
\hline SNP & Mutation & Location & Chr. position & Nucleotide change & Haplotype 1 & Haplotype 2 & Haplotype 3 \\
\hline Rs12470143 & & Intron 1 & 31763558 & $\mathrm{C}>\mathrm{T}$ & $\mathrm{C}$ & $\mathrm{T}$ & $\mathrm{C}$ \\
\hline Rs12470196 & & Intron 1 & 31763752 & $\mathrm{C}>\mathrm{T}$ & C & $\mathrm{T}$ & $\mathrm{C}$ \\
\hline Rs57971483 & & Intron 1 & 31765510 & $\mathrm{C}>\mathrm{T}$ & $\mathrm{T}$ & $\mathrm{C}$ & $\mathrm{T}$ \\
\hline Rs4952220 & & Intron 1 & 31765556 & $\mathrm{~A}>\mathrm{C}$ & C & A & $\mathrm{C}$ \\
\hline Rs2300697 & & Intron 1 & 31786637 & $\mathrm{C}>\mathrm{T}$ & C & $\mathrm{T}$ & $\mathrm{T}$ \\
\hline Rs2300698 & & Intron 1 & 31786793 & $A>G$ & A & G & G \\
\hline Rs2300699 & & Intron 1 & 31786967 & $\mathrm{G}>\mathrm{T}$ & G & $\mathrm{T}$ & $\mathrm{T}$ \\
\hline Rs2300700 & & Intron 1 & 31786992 & $A>G$ & G & A & G \\
\hline Rs2300701 & & Intron 1 & 31787008 & $A>G$ & A & G & G \\
\hline Rs522638 & & Intron 1 & 31805675 & $A>G$ & A & G & G \\
\hline Rs523349 & & Exon 1 & 31805706 & $\mathrm{C}>\mathrm{G}$ & G & $\mathrm{C}$ & $\mathrm{C}$ \\
\hline Rs632148 & & $5^{\prime}$-UTR & 31806031 & $\mathrm{C}>\mathrm{G}$ & C & G & G \\
\hline Control (allele) & & & & & 1494 & 250 & 936 \\
\hline & p.Gln6* & Exon 1 & 31805954 & $\mathrm{C}>\mathrm{T}$ & $\mathrm{T}$ & $\mathrm{C}$ & $\mathrm{C}$ \\
\hline Case (allele) & & & & & 5 & 5 & 1 \\
\hline
\end{tabular}

nongenital skin. It is well known that dehydroepiandrosterone (DHEA) and androstenedione or androstenediol are key intermediates in the synthesis of testosterone, and fetal DHT is produced in situ in its target organ in the "classic" pathway of androgen synthesis. Recently, an alternative, or "backdoor," biosynthetic pathway was reported to exist, leading to the production of DHT in the fetal testis, in which progesterone was converted to $5 \alpha$-dihydroprogesterone by SRD5A1 and $3 \alpha$-hydroxysteroid dehydrogenase (aldo-keto reductase $(\mathrm{AKR}))[18,19]$. The finding that human $A K R 1 C 2$ mutations can result in $46, \mathrm{XY}$ DSD shows that DHT produced in the fetal testis via the backdoor pathway acts as a hormone that collaborates with DHT produced in the genital skin by SRD5A2 to induce labioscrotal fusion [19].

In patients 1 and 2 , they all have three mutations. In the first patient, a cloning experiment indicated that p.Gln6*and p.Lys35Asn were located in the same chromosome. Due to the severe truncating mutation at codon 6 (p.Gln6*), the latter mutation (p.Lys35Asn) could not be expressed in the mature protein. So, p.Gln6* and p.Phe234Leu were pathogenic mutations in this patient. In the second patient, p.Gly203Ser and p.Arg227Gln were located in different chromosomes. We cannot perform a cloning test because of the long distance between p.Gly34Arg and p.Gly203Ser/p.Arg227Gln on the chromosome. In a previous study, functional analysis showed that p.Gly34Arg only retained about $1.2 \%$ of the enzyme activity [20]. So, all three mutations caused inactivity of the enzyme in the second patient.

To be noted, 17 (68\%) out of 25 patients were raised initially as females in our study. Of these 17 patients, 16 (94.1\%) changed their social gender to male. However, the ratios of female social sex and female-to-male sex change range from 77 to $93 \%$ and 12 to $50 \%$, respectively, in Brazilian and French studies [21]. In a Chinese study, Song et al. analyzed 86 children (age 2 months to 17 years) with $5 \alpha$-reductase deficiency and found that $27(31.4 \%)$ out of the 86 patients were raised as females [22]. Cheng et al. found $4(8.9 \%)$ out of 45 Chinese patients (age 3 months to 12 years) were raised as females [23]. These two Chinese studies did not refer to the female-to-male social sex change. The female social sex ratio of our patients was similar to that in a French study [24] but was higher than those in two Chinese studies. However, the ratio of female-to-male sex change in our study was obviously higher than those in Brazilian and French studies. This might be attributed to the age of diagnosis [21]. In the Brazilian and French studies, the patients were diagnosed at an average age of 16 and 7.6 years old, respectively. Thus, the prevalence of social sex alteration in the Brazilian study was higher than that in the French study (50\% vs. $12 \%)$. In our study, 19 patients (76\%) were older than 16 years and the average age at diagnosis was 18 years. So the ratio of social sex change was higher than the Brazilian study. Besides that, most of our patients were from remote areas (such as Guizhou and Jiangxi) and presented with female external genitalia. Poor medical conditions and insufficient attention of parents might also be considered.

In our study, p.Gly203Ser and p.Gln6* were the most common mutations. p.Gly203Ser was identified in 8 patients with an allele frequency $20.8 \%$ and was homozygous in nonconsanguineous patients 4 and 10. Zhang et al. [25] identified the heterozygous mutation p.Gly203Ser in 3 patients and presumably suggested a founder effect in the Chinese population. However, in our previous study [9], we found that p.Gly203Ser in SRD5A2 was the most prevalent mutation and that there was no founder effect of this mutation in the Chinese population. In this study, seven patients from 6 unrelated families were heterozygous for the nonsense mutation p.Gln6*, which was reported mainly in East Asia.

For haplotype analysis, we selected 12 SNPs near p.Gln6* based on the genotyping data from 1468 Chinese Han individuals in GWAS data [16]. Actually, these 12 SNPs could form more than 3 haplotypes, but we only listed 3 haplotypes which were found in 6 patients with p.Gln $6 *$ mutation in Table 4. Mutant allele T (p.Gln6*) could be found both in haplotypes 1 ( 5 alleles) and 3 ( 1 alleles). Moreover, wild genotype $\mathrm{C}$ and mutant genotype $\mathrm{T}$ simultaneously presented in haplotype 3 . So, there was no linkage disequilibrium between 
p.Gln6* and the other 12 SNPs. Besides, no geographic relationship was found in 5 patients who shared haplotype 1 . Therefore, p.Gln6* does not appear to derive from a common ancestor in the Chinese population, which is consistent with the fact that it has been reported in Mexico, China, Korea, and Thailand $[8,9,13,26]$. The recurrence of a particular mutation in a geographically isolated region suggests a founder effect; for example, two specific CYP17A1 mutations in China or East Asia have been rarely reported in other ethnic groups [27, 28]. Similarly, two population-specific mutations in CYP17A1 (p.Trp406Arg and p.Arg362Cys) also showed a founder effect in Brazil [29].

The p.Arg246Gln substitution that we found in patients $3,17,18$, and 19 seems to be a hot spot of SRD5A2 mutations since it has been reported in patients with diverse geographic and ethnic backgrounds, including those with Korean, Indian, Mexican, and Italian ethnic backgrounds [13, 30-32]. Eunice et al. [33] even proposed a founder gene effect of this mutation in India. Functional studies of this mutation suggested that it decreases the affinity for the cofactor NADPH $[1,34]$. The optimal $\mathrm{pH}$ of the mutant enzyme is also changed, resulting in a disruption of the activity of the enzyme. Missense mutations that result in less than $0.4 \%$ activity of the $5 \alpha$-reductases usually result in ambiguous or female external genitalia [26]. p.Arg227Gln was also detected in four patients (patients 2, 10, 13, and 15). Cheng et al. [23] reported that the p.Arg227Gln mutation was most frequently reported $(91.1 \%)$ in South China, which suggested a founder effect. However, the incidence of p.Arg227Gln was relatively lower in our cohort (4/25).

There were some less frequent mutations detected in our study, such as p.Gly34Arg and c.182-2A > G. p.Gly34Arg was identified only in one patient (patient 2). However, this mutation was prevalent in Egypt and had linkage disequilibrium with the V89L polymorphism, which indicated a founder effect of the p.Gly34Arg mutation among Egyptians [35]. A c.182-2 A $>\mathrm{G}$ mutation was detected in a set of siblings (patients 21 and 22); this mutation is common in GreekCypriot patients with $5 \alpha$-reductase deficiency and is very likely to be the result of a founder effect [36, 37]. Vilchis et al. [38] analyzed 11 Mexican patients with steroid $5 \alpha$-reductase 2 deficiency. They found that $40 \%$ of the mutant alleles (9/22) contained the gene variant p.Pro212Arg and hypothesized that the presence of this mutation may constitute a founder gene effect. However, we did not discover this mutation in our cohort.

\section{Conclusion}

Eighteen mutations were identified in 25 Chinese patients with $5 \alpha$-RD2 deficiency. p.Gln6* and p.Gly203Ser were the most prevalent mutations. Although no homozygous p.Gln6* mutation and only two homozygous p.Gly203Ser mutations were included, the patients with or without p.Gln6* and p.Gly203Ser did not demonstrate a significant difference in hormone levels. Finally, no founder effect of the p.Gln6* mutation in the SRD5A2 gene was detected in the Chinese population.

\section{Abbreviations}

SNPs: Single-nucleotide polymorphisms

5 $\alpha$-RD2: $5 \alpha$-Reductase type 2

DHT: Dihydrotestosterone

GWAS: Genome-wide association study.

\section{Data Availability}

Data are available upon request to corresponding author.

\section{Ethical Approval}

This study was approved by the Ethics Committee of Shanghai Ninth People's Hospital.

\section{Consent}

Written informed consent was obtained from all the adult patients themselves or from the parents of child patients.

\section{Conflicts of Interest}

The authors declare that they have no conflicts of interest.

\section{Authors' Contributions}

JQ and HY designed and supervised this investigation. $\mathrm{BH}$ and TC performed this investigation. $\mathrm{HZ}$ and $\mathrm{WZ}$ contributed to the data collection. HS and JY provided technical or material support. All authors read and approved the final manuscript. Bing Han and Tong Cheng contributed equally to this work.

\section{Acknowledgments}

We are grateful to all the patients who participated in the study. The article was revised by AJE. This study is supported by grants from the National Nature Science Foundation of China (Nos. 81873652, 81570753, and 81670717), the Shanghai Committee of Science and Technology (No. 15ZR1425000), and the Clinical Research Project of Multidisciplinary Team, Shanghai Ninth People's Hospital, Shanghai JiaoTong University School of Medicine (201903).

\section{References}

[1] A. E. Thigpen, D. L. Davis, A. Milatovich et al., "Molecular genetics of steroid 5 alpha-reductase 2 deficiency," The Journal of Clinical Investigation, vol. 90, no. 3, pp. 799-809, 1992.

[2] A. E. Thigpen, R. I. Silver, J. M. Guileyardo, M. L. Casey, J. McConnell, and D. W. Russell, "Tissue distribution and ontogeny of steroid 5 alpha-reductase isozyme expression," The Journal of Clinical Investigation, vol. 92, no. 2, pp. $903-$ 910, 1993.

[3] M. Maes, C. Sultan, N. Zerhouni, S. W. Rothwell, and C. J. Migeon, "Role of testosterone binding to the androgen receptor in male sexual differentiation of patients with 5 alphareductase deficiency," Journal of Steroid Biochemistry, vol. 11, no. 4, pp. 1385-1392, 1979. 
[4] J. Imperato-McGinley, L. Guerrero, T. Gautier, J. L. German, and R. E. Peterson, "Steroid 5alpha-reductase deficiency in man. An inherited form of male pseudohermaphroditism," Birth Defects Original Article Series, vol. 11, no. 4, pp. 91-103, 1975.

[5] P. C. Walsh, J. D. Madden, M. J. Harrod, J. L. Goldstein, P. MacDonald, and J. D. Wilson, "Familial incomplete male pseudohermaphroditism, type 2. Decreased dihydrotestosterone formation in pseudovaginal perineoscrotal hypospadias," The New England Journal of Medicine, vol. 291, no. 18, pp. 944-949, 1974.

[6] S. H. Kim, K. S. Kim, G. H. Kim, B. M. Kang, and H. W. Yoo, "A novel frameshift mutation in the $5 \alpha$-reductase type 2 gene in Korean sisters with male pseudohermaphroditism," Fertility and Sterility, vol. 85, no. 3, pp. 750.e9-750.e12, 2006.

[7] M. Bahceci, A. R. Ersay, A. Tuzcu, O. Hiort, A. Richter-Unruh, and D. Gokalp, "A novel missense mutation of 5-alpha reductase type 2 gene (SRD5A2) leads to severe male pseudohermaphroditism in a Turkish family," Urology, vol. 66, no. 2, pp. 407-410, 2005.

[8] T. Sahakitrungruang, S. Wacharasindhu, P. Yeetong, T. Snabboon, K. Suphapeetiporn, and V. Shotelersuk, "Identification of mutations in the SRD5A2 gene in Thai patients with male pseudohermaphroditism," Fertility and Sterility, vol. 90, no. 5, pp. 2015.e11-2015.e15, 2008.

[9] H. Zhu, W. Liu, B. Han et al., "Phenotypic and molecular characteristics in eleven Chinese patients with $5 \alpha$-reductase type 2 deficiency," Clinical Endocrinology, vol. 81, no. 5, pp. 711-720, 2014.

[10] I. Mazen, Y. Z. Gad, M. Hafez, C. Sultan, and S. Lumbroso, "Molecular analysis of 5alpha-reductase type 2 gene in eight unrelated Egyptian children with suspected 5alpha-reductase deficiency: prevalence of the G34R mutation," Clinical Endocrinology, vol. 58, no. 5, pp. 627-631, 2003.

[11] E. M. Costa, S. Domenice, M. H. Sircili, M. Inacio, and B. B. Mendonca, "DSD due to $5 \alpha$-reductase 2 deficiency-from diagnosis to long term outcome," Seminars in Reproductive Medicine, vol. 30, no. 5, pp. 427-431, 2012.

[12] C. K. Cheon, "Practical approach to steroid 5alpha-reductase type 2 deficiency," European Journal of Pediatrics, vol. 170, no. 1, pp. 1-8, 2011.

[13] J. M. Ko, C. K. Cheon, G. H. Kim, S. H. Kim, K. S. Kim, and H. W. Yoo, "Clinical characterization and analysis of the SRD5A2 gene in six Korean patients with 5alpha-reductase type 2 deficiency," Hormone Research in Poediatrics, vol. 73, no. 1, pp. 41-48, 2010.

[14] T. Cheng, H. Wang, B. Han et al., "Identification of three novel SRD5A2 mutations in Chinese patients with $5 \alpha$-reductase 2 deficiency," Asian Journal of Andrology, vol. 21, no. 6, pp. 577-581, 2019.

[15] S. F. Ahmed, O. Khwaja, and I. A. Hughes, "The role of a clinical score in the assessment of ambiguous genitalia," BJU International, vol. 85, no. 1, pp. 120-124, 2000.

[16] X. Chu, C. M. Pan, S. X. Zhao et al., "A genome-wide association study identifies two new risk loci for Graves' disease," Nature Genetics, vol. 43, no. 9, pp. 897-901, 2011.

[17] J. D. Wilson, J. E. Griffin, and D. W. Russell, "Steroid 5 alphareductase 2 deficiency," Endocrine Reviews, vol. 14, no. 5, pp. 577-593, 1993.

[18] C. E. Flück, M. Meyer-Böni, A. V. Pandey et al., "Why boys will be boys: two pathways of fetal testicular androgen biosyn- thesis are needed for male sexual differentiation," American Journal of Human Genetics, vol. 89, no. 2, pp. 201-218, 2011.

[19] R. J. Auchus, "The backdoor pathway to dihydrotestosterone," Trends in Endocrinology and Metabolism, vol. 15, no. 9, pp. 432-438, 2004.

[20] N. M. Makridakis, E. di Salle, and J. K. Reichardt, "Biochemical and pharmacogenetic dissection of human steroid 5 alphareductase type II," Pharmacogenetics, vol. 10, no. 5, pp. 407413,2000

[21] B. B. Mendonca, R. L. Batista, S. Domenice et al., "Steroid $5 \alpha$-reductase 2 deficiency," The Journal of Steroid Biochemistry and Molecular Biology, vol. 163, pp. 206-211, 2016.

[22] Y. N. Song, L. J. Fan, X. Zhao, and C. X. Gong, "Clinical phenotype and gene analysis of 86 cases of 5 alpha reductase deficiency," Zhonghua Er Ke Za Zhi, vol. 57, no. 2, pp. 131135,2019 .

[23] J. Cheng, R. Lin, W. Zhang et al., "Phenotype and molecular characteristics in 45 Chinese children with $5 \alpha$-reductase type 2 deficiency from South China," Clinical Endocrinology, vol. 83, no. 4, pp. 518-526, 2015.

[24] L. Maimoun, P. Philibert, B. Cammas et al., "Phenotypical, biological, and molecular heterogeneity of $5 \alpha$-reductase deficiency: an extensive international experience of 55 patients," The Journal of Clinical Endocrinology and Metabolism, vol. 96, no. 2, pp. 296-307, 2011.

[25] M. Zhang, J. Yang, H. Zhang, G. Ning, X. Li, and S. Sun, “A novel SRD5A2 mutation with loss of function identified in Chinese patients with hypospadias," Hormone Research in Pediatrics, vol. 76, no. 1, pp. 44-49, 2011.

[26] P. Canto, F. Vilchis, B. Chávez et al., "Mutations of the 5 alphareductase type 2 gene in eight Mexican patients from six different pedigrees with 5 alpha-reductase-2 deficiency," Clinical Endocrinology, vol. 46, no. 2, pp. 155-160, 1997.

[27] J. Yang, B. Cui, S. Sun et al., "Phenotype-genotype correlation in eight Chinese 17alpha-hydroxylase/17,20 lyase-deficiency patients with five novel mutations of CYP17A1 gene," The Journal of Clinical Endocrinology and Metabolism, vol. 91, no. 9, pp. 3619-3625, 2006.

[28] J. Qiao, X. Chen, C.-L. Zuo et al., "Identification of steroid biosynthetic defects in genotype-proven heterozygous individuals for 17alpha-hydroxylase/17,20-lyase deficiency," Clinical Endocrinology, vol. 72, no. 3, pp. 312-319, 2010.

[29] M. Costa-Santos, C. E. Kater, and R. J. Auchus, "Two PrevalentCYP17Mutations and Genotype-Phenotype Correlations in 24 Brazilian Patients with 17-Hydroxylase Deficiency," The Journal of Clinical Endocrinology \& Metabolism, vol. 89, pp. 49-60, 2004.

[30] A. Nicoletti, L. Baldazzi, A. Balsamo et al., "SRD5A2 gene analysis in an Italian population of under-masculinized 46,XY subjects," Clinical Endocrinology, vol. 63, no. 4, pp. 375-380, 2005.

[31] R. Sahu, R. Boddula, P. Sharma et al., "Genetic analysis of the SRD5A2 gene in Indian patients with 5alpha-reductase deficiency," Journal of Pediatric Endocrinology \& Metabolism, vol. 22, no. 3, pp. 247-254, 2009.

[32] F. Vilchis, J. P. Méndez, and P. Canto, "Identification of missense mutations in the SRD5A2 gene from patients with steroid $5 \alpha$-reductase 2 deficiency," Clinical Endocrinology, vol. 52, no. 3, pp. 383-387, 2000. 
[33] M. Eunice, P. Philibert, B. Kulshreshtha et al., "Molecular diagnosis of 5alpha-reductase-2 gene mutation in two Indian families with male pseudohermaphroditism," Asian Journal of Andrology, vol. 10, no. 5, pp. 815-818, 2008.

[34] Y. Wang, Q. Li, J. Xu et al., "Mutation analysis of five candidate genes in Chinese patients with hypospadias," European Journal of Human Genetics, vol. 12, no. 9, pp. 706-712, 2004.

[35] Y. Z. Gad, R. Khairt, I. Mazen, and H. G. Osman, "Detection of the G34R mutation in the 5 alpha reductase 2 gene by allele specific PCR and its linkage to the 89L allele among Egyptian cases," Sexual Development, vol. 1, no. 5, pp. 293-296, 2007.

[36] N. Skordis, C. Shammas, E. Efstathiou, A. Sertedaki, V. Neocleous, and L. Phylactou, "Late diagnosis of 5alpha steroid-reductase deficiency due to IVS12A $>$ G mutation of the SRD5a2 gene in an adolescent girl presented with primary amenorrhea," HORMONES, vol. 10, no. 3, pp. 230-235, 2011.

[37] N. Skordis, V. Neocleous, A. Kyriakou et al., "The IVS1-2A>G mutation in the SRD5A2 gene predominates in Cypriot patients with $5 \alpha$ reductase deficiency," Journal of Endocrinological Investigation, vol. 33, no. 11, pp. 810-814, 2010.

[38] F. Vilchis, L. Ramos, J. P. Mendez, S. Benavides, P. Canto, and B. Chavez, "Molecular analysis of the SRD5A2 in 46,XY subjects with incomplete virilization: the P212R substitution of the steroid 5alpha-reductase 2 may constitute an ancestral founder mutation in Mexican patients," Journal of Andrology, vol. 31, no. 4, pp. 358-364, 2010. 